\title{
Multicast Source Handover Scheme based on Proxy Router Discovery
}

\author{
Takeshi Takahashi*, Koichi Asatani ${ }^{\ddagger *}$, Hideyoshi Tominaga* \\ * Graduate School of Global Information and Telecommunication Studies, Waseda University, Tokyo, 169-8555 Japan \\ Email: \{take,tominaga\}@ tom.comm.waseda.ac.jp \\ $\ddagger$ Graduate School of Electrical and Electronic Engineering, Kogakuin University, Tokyo, 163-8677 Japan \\ Email: asatanik@cc.kogakuin.ac.jp
}

\begin{abstract}
To support multicast over Mobile IP networks, the IETF proposed two schemes, namely bi-directional tunneling (MIP-BT) and remote subscription (MIP-RS). MIP-BT provides mobility by establishing bi-directional tunnel from home agent to mobile node's care-of address. Although it does not require multicast tree reconstruction, it suffers from redundant routing that causes packet delivery delay and network bandwidth consumption. On the other hand, MIP-RS provides mobility by reconstructing multicast tree when mobile node enters a foreign network. Although MIP-RS provides shortest multicast routing path, it requires the reconstruction of entire multicast tree in case of source mobility and causes service disruption time during the process of handover. To cope with those problems, we propose new source mobility scheme that enjoys the advantages of MIPRS and provides seamless handover with the help of proxy router, which assists handover by swapping network addresses and conducting packet forwarding. We examine the deployment issues of the proposed scheme over the Internet and evaluate the proposed scheme from the viewpoint of routing distance and network bandwidth consumption through simulation experiments with various parameters, and then clarify the effectiveness and efficiency of the proposed scheme.
\end{abstract}

\section{INTRODUCTION}

As a technical standard that supports mobility over IP networks, Mobile IP version 6 (Mobile IPv6) [1], [2] is standardized by the Internet Engineering Task Force (IETF). It specifies the operation of the IPv6 [3] Internet with mobile nodes (MNs). Each $\mathrm{MN}$ is always identified by its home address regardless of its current point of attachment to the Internet. Whilst situated away from its home, an $\mathrm{MN}$ is also associated with a care-of address (CoA), which provides information about the MN's current location. IPv6 packets addressed to an MN's home address are transparently routed to its CoA [4].

On the other hand, due to the development of the broadband network infrastructure, multimedia content delivery services such as video streaming services and IPTV services have got a grip on reality and some of them are already started. Multicast is the required technologies to efficiently provide one-to-many communication and is expected to take great roles in those multimedia content delivery services. However, the current multicast protocols such as DVMRP [5], MOSPF [6], CBT [7], and PIM [8] are not designed for mobile communications. Hence, further protocol enhancements are required for efficient multicast support over Mobile IP networks.
Although there are several multicast routing protocols, this paper focuses on PIM-SM [8] with shortest-path tree(SPT). PIM-SM can utilize core-based trees (CBTs) and SPTs by adopting a tree switching mechanism. The tree switching mechanism allows receivers to establish the SPT from the senders with high-bit-rate traffic apart from the established shared tree for the group. In general, a CBT multicast architecture has scalability problems caused by traffic concentration on its core router and shared multicast tree. If the network grows larger, traffic may be concentrated too intensively on the shared tree. As a result, network congestion is easily induced, and network performance worsens [9]. Moreover, the routing path from source to receiver is not optimal in case of CBT multicasting. In the forthcoming broadband content communication era, redundant routing increases the network resource consumption dramatically. Hence, we believe the support of SPT is mandatory. Hence, this paper focuses on PIM-SM network with SPT.

To support multicast over Mobile IP networks, the IETF proposed two different schemes, namely bi-directional tunneling (MIP-BT) and remote subscription (MIP-RS). In MIP-BT, when an $\mathrm{MN}$ is visiting foreign network, it establishes bidirectional tunnel with home agent (HA). Hence, multicast traffic sent to HA will be forwarded to the $\mathrm{MN}$ via the tunnel regardless of the current location of the MN. Multicast traffic sent from $\mathrm{MN}$ is also forwarded to multicast receivers via HA by utilizing the tunnel regardless of the current location of the MN. Although this scheme hides MN's mobility and avoids the reconstruction of multicast tree, the multicast packet delivery path becomes redundant and reduces the benefits of multicasting.

In MIP-RS, when an $\mathrm{MN}$ is visiting foreign network, it establishes multicast tree from the new network. Hence, HA is not required to establish tunnel, and the multicast routing path will be the shortest. However, this scheme suffers from the multicast tree reconstruction delay, which causes service disruption and out-of-synch problem. In case of source mobility, this scheme requires the reconstruction of entire multicast tree and causes service disruption time during the process of handover.

To cope with those problems, we focus on source mobility and propose new scheme that enjoys the advantages of MIPRS and provides seamless handover with the help of proxy 
router(PR), which assists handover by swapping network addresses and conducting packet forwarding. Here, the PRs are chosen for each handover dynamically. Moreover, our proposed scheme is designed to be compatible with Fast Handovers for Mobile IPv6(FMIP) [10] with proper enhancement, with which the service disruption time can be minimized. This paper also discusses on the deployment issues of the proposed scheme over the Internet. Simulation experiments evaluate the proposed scheme from the viewpoint of routing distance and network bandwidth consumption with various parameters, and clarify the effectiveness and efficiency of the proposed scheme.

\section{RELATED WORKS}

To support multicast over Mobile IP, plenty of researches have been proposed until now [9], [11-22], and most of them can be categorized as extensions to either MIP-BT or MIPRS. This section describes the mobility support schemes over MIP-BT and MIP-RS.

\section{A. Mobility Support over MIP-BT and its Extensions}

In MIP-BT, an MN subscribes to multicast groups through its HA. When the MN is visiting foreign network, it establishes bi-directional tunnel with its HA. All the multicast packets will be routed via the HA through the bi-directional tunnel.

In case of receiver mobility, it suffers from duplicate packet forwarding. Assuming multiple MNs that subscribe to the same multicast group are visiting one same foreign network, all of them will receive the same multicast traffic from their HAs through their own tunnels individually even though they are in the same network. Although the advantage of multicast is to suppress duplicate traffic to the same network, MIP-BT causes duplicate traffic receiving due to its tunneling feature. To solve this duplication issue, Mobile Multicast Protocol (MoM) [11] was proposed. MoM chooses designated multicast service provider (DMSP) among HAs that forwards the same traffic. By choosing single DMSP, the packet duplication can be avoided whilst MoM has known problems such as DMSP handover [11]. However, MIP-BT and MoM still suffers from redundant routing due to the fact all multicast traffic traverse through HA.

In case of source mobility, all packets are sent to its HA and then forwarded to the multicast group. Hence, this scheme does not require multicast tree reconstruction in case of source mobility. However, as is in receiver mobility case, since multicast packets are always delivered via HA, the routing path is redundant. Especially when the source is moving far away from its home network, the routing redundancy will be significant, and the HA and its surrounding network resources will be the bottle-neck of communications.

\section{B. Mobility Support over MIP-RS and its Extensions}

MIP-RS is another approach to support mobility over Mobile IP networks. In this scheme, an MN subscribes to multicast groups when it visits foreign network, and it receives multicast packets directly from the closest multicast router without any tunneling. Although this scheme provides shortest multicast routing path, it causes several drawbacks.

In case of receiver mobility, since the multicast packet delivery is not synchronized, when MN moves from one multicast router to another, the traffic will be discontinuous. Moreover, if the visiting network is not the member of multicast group, the access router (AR) is required to subscribe to the interested multicast group. To cope with receiver mobility problem of MIP-RS, several schemes [12-18] have been proposed. Those proposals typically perform packet forwarding from MN's previous network to new network and solve the out-of-synch problem of MIP-RS.

In case of source mobility, MIP-RS and those schemes mentioned in [12-19] provide no solution other than MIP-BT. Practically, MIP-RS and its extension require the reconstruction of the entire multicast tree and cause significant service disruption time. Hence, utilizing MIP-BT for source mobility has been preferred to MIP-RS. However, the advantage of utilizing MIP-RS, i.e. shortest multicast forwarding path, is very obvious even in case of source mobility. There are two remarkable schemes for source mobility based on MIP-RS: source mobility support multicast (SMM) [20] and mobile SSM source (MSSMS) [21].

SMM suppresses the overhead of multicast tree reconstruction in case of source mobility. SMM is based on a concept where multicast tree is constructed over Cellular IP network [23], in which a shortest path tree (SPT) and rendezvous point tree (RPT) are combined into one multicast tree. However, SMM is based on Cellular IP and is required some enhancements to be adapted to the other IP networks.

MSSMS is a scheme to support Source-Specific Multicast (SSM) over Mobile IPv6 networks by re-establishing multicast tree. When a source moves into a new subnet, it announces the multicast receivers about its new $\mathrm{CoA}\left(\mathrm{CoA}_{i+1}\right)$. To ensure consistency at higher layers, i.e. transport layer, the announcement must also indicate the home address of the source to notify applications about the home address of the mobile source. Receivers subsequently subscribe to the new multicast group $\left(\mathrm{CoA}_{i+1}, \mathrm{G}\right)$. Although the construction of new multicast tree requires some time, by manipulating old multicast tree and new multicast tree, receivers can receive multicast traffic smoothly. During the process of handover, i.e. during the construction of new multicast tree, the source sends multicast packets to previous $\mathrm{AR}\left(\mathrm{AR}_{i}\right)$ with IP encapsulation, and then the $\mathrm{AR}_{i}$ decapsulates them and forwards them to $\left(\mathrm{CoA}_{i}, \mathrm{G}\right)$ multicast tree as can be seen in Fig. 1 .

Although this scheme enables smooth transition from $\left(\mathrm{CoA}_{i}, \mathrm{G}\right)$ multicast tree to $\left(\mathrm{CoA}_{i+1}, \mathrm{G}\right)$ multicast tree, it suffers from redundant routing that causes packet routing delay and network bandwidth consumption during the process of handover. For instance, in Fig. 1, although Receiver 3 is only 3 hops away from new $\mathrm{AR}\left(\mathrm{AR}_{i+1}\right)$, the actual routing distance from $\mathrm{AR}_{i+1}$ to Receiver 3 is 9 hops since the multicast traffic must be forwarded via $\mathrm{AR}_{i}$.

In our proposed scheme, we reduce the redundant routing during the process of handover and provide more efficient 


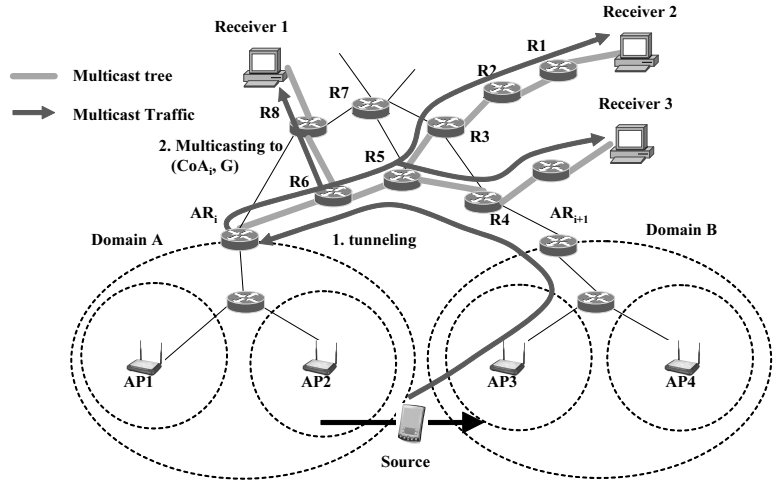

Fig. 1. Redundant routing during the process of handover

smooth handover from the viewpoint of routing distance and network bandwidth consumption.

\section{PRoposed SCHEME}

Since MIP-RS has the advantage of the shortest path route compared to MIP-BS, it is very beneficial to have source mobility protocol based on MIP-RS if we can improve the performance during the process of handover. The advantage is even more obvious when MNs move far away from their home networks. During the process of handover, due to the reconstruction of the entire multicast tree, MIP-RS causes serious service disruption time. MSSMS [21] avoids this service disruption problem by manipulating two multicast trees, i.e. $\left(\mathrm{CoA}_{i}, \mathrm{G}\right)$ tree and $\left(\mathrm{CoA}_{i+1}, \mathrm{G}\right)$ tree. However, it suffers from redundant routing that causes long routing distance and network bandwidth consumption during the process of handover. In case of broadband content delivery services, it causes excess network resource consumptions and may causes network congestion and bottle-neck temporarily. To cope with this problem, we propose new scheme that enjoys the advantages of MIP-RS and provides seamless handover with the help of PR, which assists handover by swapping network addresses of the multicast packets received from $\left(\mathrm{AR}_{i+1}, \mathrm{G}\right)$ tree and forward the packets to $\left(\mathrm{AR}_{i}, \mathrm{G}\right)$ tree. Although our proposed scheme is designed for PIM-SM with SPTs, it can be adapted to the other multicast protocols such as PIM-SSM [24] with proper enhancements.

Different from MSSMS, our proposed scheme utilizes AR's address instead of CoA as a source address of multicast traffic. As we describe later in this section, PRs are required to subscribe to new multicast group before an $\mathrm{MN}$ is sending multicast traffic from new network. However, the MN cannot obtain $\mathrm{CoA}_{i+1}$ before moving to next network without the full support of FMIP. Moreover, FMIP also has possibility to fail to provide $\mathrm{CoA}_{i+1}$ information before moving to new network. Hence, we utilize AR's address instead of CoA as a source of multicast traffic so that new multicast tree can be established before the $\mathrm{MN}$ moves into the new network regardless of whether the MN succeed in obtaining $\mathrm{CoA}_{i+1}$.

In this paper, the multicast router that can work as PR if re- quested is termed PR candidate whilst the multicast router that cannot work as PR is termed as non-protocol multicast router. Our proposed scheme assumes that ARs support multicast and our proposed protocol, and that ARs will be the Designated Routers for MNs. If the AR does not support our proposed protocol, the handover procedure described in MSSMS should be taken.

Figure 2 and Fig. 3 show the procedure of our proposed scheme. The proposed scheme can be divided into two phases, namely, handover initiation phase and transient routing.

\section{A. Handover Initiation Phase}

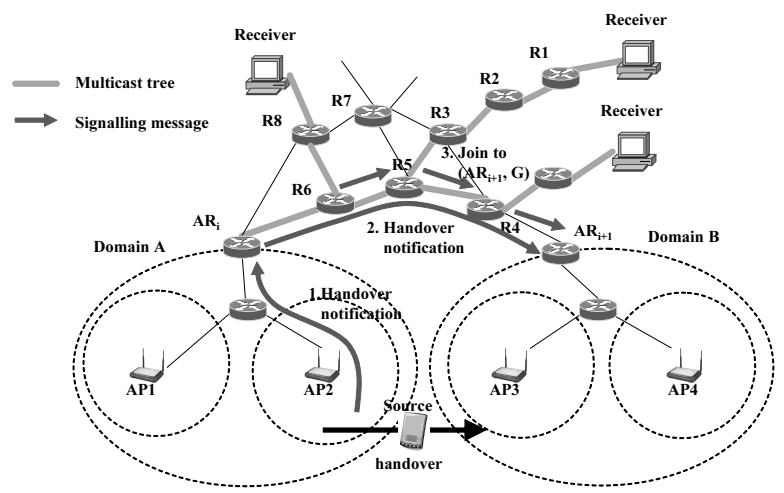

Fig. 2. Handover initiation

An MN starts handover procedure by sending Handover notification message to its $\mathrm{AR}_{i}$ just before switching its link to new network. This message includes the information of next access point (AP) identifier. This procedure can be substituted for FBU of FMIP [10] if FMIP is supported. Upon receiving the Handover notification message, the $\mathrm{AR}_{i}$ retrieves the $\mathrm{AR}_{i+1}$ address from its own local database by utilizing next AP identifier information as a key. Note that each AR is assumed to have the database of its geographically neighboring ARs' address information and their APs' information, and is assumed to be able to retrieve the $\mathrm{AR}_{i+1}$ address by looking up the AP identifier information. This database can be created manually or by performing periodic message exchanges between its geographically neighboring ARs as is also seen in FMIP though the details are outside the scope of this paper. Upon receiving the Handover notification message, the $\mathrm{AR}_{i}$ appends hop-by-hop option of IPv6 [3] inside the message and forwards it to $\mathrm{AR}_{i+1}$. The $\mathrm{PR}$ candidates en route from $\mathrm{AR}_{i}$ to $\mathrm{AR}_{i+1}$ intercept the message and forward it to the next hop router toward $\mathrm{AR}_{i+1}$. If PR candidate that received the message has $\left(\mathrm{AR}_{i}, \mathrm{G}\right)$ receivers, it subscribes to the $\left(\mathrm{AR}_{i+1}, \mathrm{G}\right)$ multicast group and becomes PR. However, if the PR candidate has $\left(\mathrm{AR}_{i}, \mathrm{G}\right)$ receivers only toward the interface that is connected to another PR candidate en route to $\mathrm{AR}_{i+1}$, it stops working as PR. Note that the PR candidates can intercept the message simply by inspecting the hop-byhop option inside the message, and non-protocol multicast routers simply forward the message. When $\mathrm{AR}_{i+1}$ receives 
the Handover notification message, it establishes the state for the multicast tree $\left(\mathrm{AR}_{i+1}, \mathrm{G}\right)$ and awaits the arrival of the $\mathrm{MN}$ to its network.

\section{B. Transient Routing Phase}

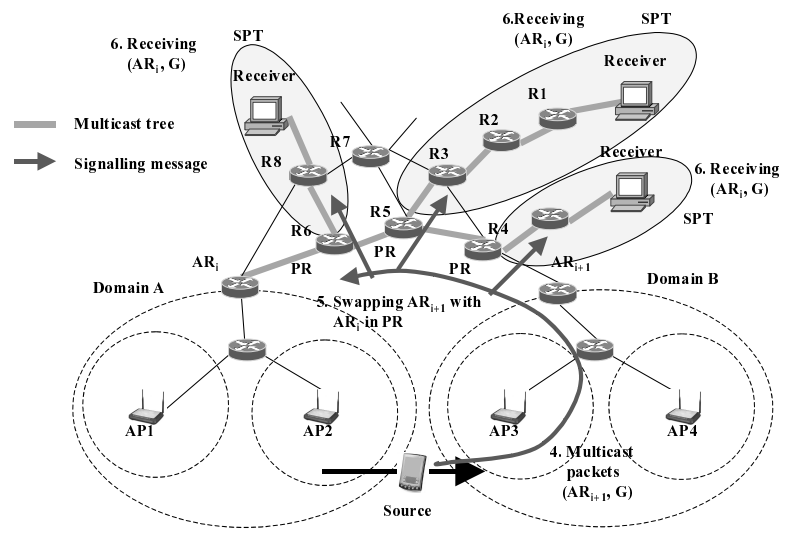

Fig. 3. multicasting during the process of handover

When the $\mathrm{MN}$ moves into the new network, i.e. $\mathrm{AR}_{i+1}$, and acquires $\mathrm{CoA}_{i+1}$, it starts sending multicast traffic to its $\mathrm{AR}_{i+1}$ preceded by Handover notification message. If the $\mathrm{AR}_{i+1}$ has already received Handover notification message before, the message will be just ignored and discarded; otherwise, the procedure described later in Fig. 5 will be conducted. Note that this Handover notification message can be easily integrated with Fast Neighbor Advertisement (FNA) message defined in FMIP.

Upon receiving multicast traffic, the $\mathrm{AR}_{i+1}$ starts forwarding the multicast traffic to the $\left(\mathrm{AR}_{i+1}, \mathrm{G}\right)$ multicast group. At this moment, the members for the tree $\left(\mathrm{AR}_{i+1}, \mathrm{G}\right)$ should be only the PRs. Note that $\mathrm{AR}_{i+1}$ itself can be PR.

Upon receiving the multicast traffic from $\left(\mathrm{AR}_{i+1}, \mathrm{G}\right), \mathrm{PR}$ swaps $\mathrm{AR}_{i+1}$ with $\mathrm{AR}_{i}$ in the source address field of the multicast packet and forwards the traffic toward its interface connected to the $\left(\mathrm{AR}_{i}, \mathrm{G}\right)$ multicast tree except the interfaces that is directly connected to other PRs. In this way, all the multicast receivers in $\left(\mathrm{AR}_{i}, \mathrm{G}\right)$ can receive traffic from $\left(\mathrm{AR}_{i+1}, \mathrm{G}\right)$. Employing PRs does not cause multicast tree reconstruction, and the tree under each PR is SPT of $\left(\mathrm{AR}_{i}, \mathrm{G}\right)$. Moreover, employing PR gives the advantage of shorter routing path, which enjoys better performance from the viewpoint of packet delivery delay and network bandwidth consumption. Although the packet routing path is still not the optimal SPT, the redundant routing will be greatly suppressed compared to the conventional schemes during the process of handover (See Section V). Note that the multicast tree for the group $\left(\mathrm{AR}_{i}, \mathrm{G}\right)$ is SPT after PRs, and that, different from MSSMS, our proposed scheme is not required to utilize encapsulation or decapsulation at all, and MN simply sends multicast traffic to its AR.

Upon receiving traffic from the $\mathrm{MN}$, the $\mathrm{AR}_{i+1}$ may ask all the receivers to subscribe to the $\left(\mathrm{AR}_{i+1}, \mathrm{G}\right)$ multicast group by announcing $\left(\mathrm{AR}_{i+1}, \mathrm{G}\right)$ as well as the MN's home address so that higher layers such as transport layer can have consistency. Here, the $\mathrm{AR}_{i+1}$ must have know the home address of the MN that can be informed by Router Solicitation message [25] with proper option or by Fast Neighbor Advertisement message [10] though the exact scheme is outside the scope of this paper. Since the establishment of $\left(\mathrm{AR}_{i+1}, \mathrm{G}\right)$ multicast tree takes some time, it is beneficial to have our scheme as a transitional routing during the process of handover. The $\mathrm{MN}$ can also wait some time before asking all the receivers to subscribe to the $\left(\mathrm{AR}_{i+1}, \mathrm{G}\right)$ multicast group if $\mathrm{MN}$ has some possibility to go back to previous network though this feature is outside the scope of this paper.

The signaling sequences are summarized in Fig. 4. Although we focused on PIM-SM in this paper, the proposed scheme can be adopted to PIM-SSM [24] or the other SPT-based multicast routing protocols with proper modifications.

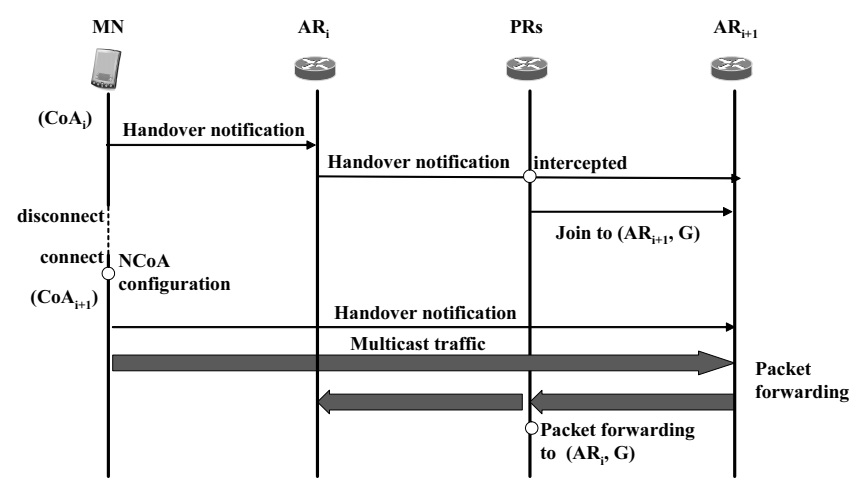

Fig. 4. Handover Sequence

It is possible that $\mathrm{AR}_{i+1}$ does not receive Handover notification message by the time the $\mathrm{MN}$ sends multicast packets. In this case, the scheme mentioned above cannot be simply applied since $\mathrm{PR}$ candidates en route from $\mathrm{AR}_{i}$ to $\mathrm{AR}_{i+1}$ are not ready to work as AR. This situation may happen in one of the following situations.

1) The MN did not send Handover notification message since it couldn't obtain the $\mathrm{AR}_{i+1}$ 's IP address. (Note that this paper generally assumes that MNs can obtain $\mathrm{AR}_{i+1}$ 's address with proper schemes such as FMIP.)

2) The MN did not send Handover notification message since it didn't realize the change of network attachment point.

3) The multicast packet sent from the $\mathrm{MN}$ reached $\mathrm{AR}_{i+1}$ reached $\mathrm{AR}_{i+1}$ prior to the Handover Notification message arrives at $\mathrm{AR}_{i+1}$

In any of these cases, the MN sends Handover notification message to $A R_{i+1}$, which in return sends Late Handover notification message to $\mathrm{AR}_{i}$. The $\mathrm{AR}_{i}$, in response to the Handover notification message, returns Handover notification message back to the $\mathrm{AR}_{i+1}$. In this way, the $\mathrm{AR}_{i+1}$ will receive at least one Handover notification message. Upon receiving the message, the procedure described above will be 
conducted.

The signaling sequences are summarized in Fig. 5.

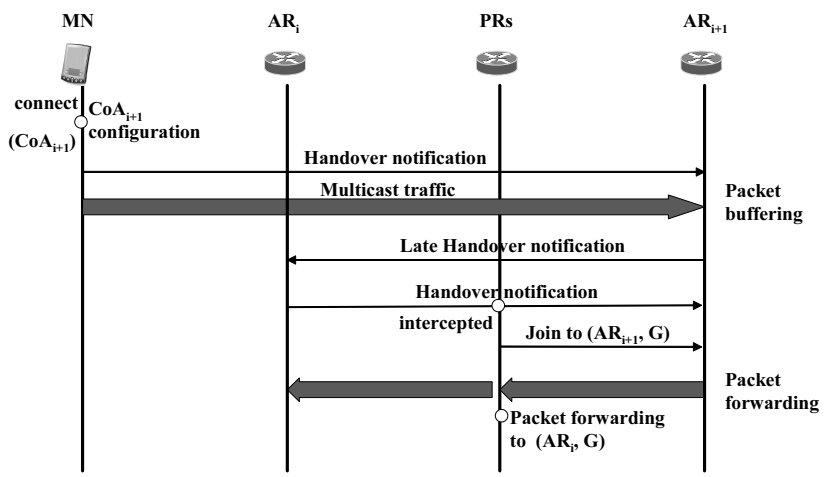

Fig. 5. Handover Sequence with Late Handover Notification Message

Note that MN sends Handover notification message in either case since it is not sure for the $\mathrm{MN}$ whether the Handover notification message sent from $\mathrm{AR}_{i}$ before its link disconnection was successfully received by $\mathrm{AR}_{i+1}$. As described above, the Handover notification message sent after the new link connection will be just discarded if the $\mathrm{AR}_{i+1}$ has already received the Handover notification message.

\section{DePloyment OVER THE INTERNET}

\section{A. Mobility and the Reverse Path Forwarding Problem}

When an MN hands over to another site, it obtains $\operatorname{CoA}_{i+1}$ to identify where it is located in the Internet. The MN directly sends its data to a multicast group. In case of multicast protocols that utilizes a packets' source such as DVMRP, MOSPF, or PIM-SM (with shortest path tree mode), multicast packets will be discarded on the IP forwarding engine in a multicast router since a MN's multicasts are expected from the link used to reach its $\mathrm{CoA}_{i}$, but when the MN moves to a new network, its datagrams will arrive on many routers via unexpected links. This problem is called Reverse Path Forwarding (RPF) Problem [9], [26]

In the proposed scheme, packets sent from the $\mathrm{MN}$ do not suffer from RPF problem. Packets sent from $\mathrm{CoA}_{i+1}$ will travel to $\mathrm{PRs}$ that locates en route from $\mathrm{AR}_{i+1}$ and $\mathrm{AR}_{i}$ according to the $\left(\mathrm{AR}_{i+1}, \mathrm{G}\right)$ multicast tree, then travel to receivers over the $\left(\mathrm{AR}_{i}, \mathrm{G}\right)$ multicast tree. Hence, multicast routers receive multicast packets from the expected links. Therefore, the proposed scheme is free from RPF problem.

\section{B. Compatibility with Non-Protocol Multicast Routers}

Our proposed scheme is compatible with non-protocol multicast routers. Even though there are non-protocol multicast routers en route from $\mathrm{AR}_{i}$ to $\mathrm{AR}_{i+1}$, our protocol works well as is shown in Fig. 6. In Fig. 6, different from Fig.3, R5 is not $\mathrm{PR}$ candidate. In this case, $\mathrm{R} 6$ that is working as PR forwards $\left(\mathrm{AR}_{i}, \mathrm{G}\right)$ multicast traffic also to $\mathrm{R} 5$ that is in the tree of $\left(\mathrm{AR}_{i}\right.$, $\mathrm{G})$ since $\mathrm{R} 5$ is a mere multicast router subscribing to $\left(\mathrm{AR}_{i}, \mathrm{G}\right)$ for R6 in this situation. As a result, as is in the case described in Section III, employing PRs does not cause multicast tree reconstruction, and the tree under each PR is SPT of $\left(\mathrm{AR}_{i}, \mathrm{G}\right)$. Although obvious redundant routing occurs between R6 and R5 in this case, by strategically locating PR candidates, the amount of redundant routing is still improved compared to MSSMS.

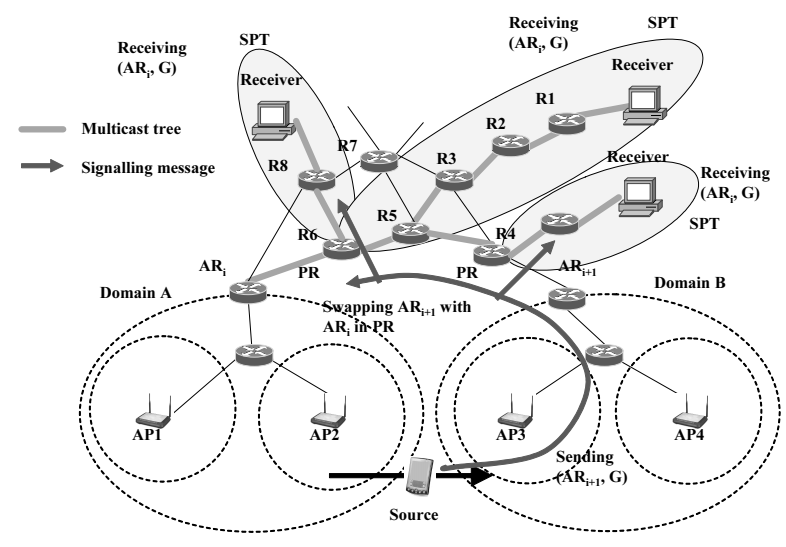

Fig. 6. Compatibility with non-protocol multicast routers

\section{Deployability}

As described in the previous section, our protocol can cooperate with existing multicast routers. Hence, PR can be deployed gradually. PR should be deployed strategically so that the proposed scheme can work efficiently though the deployment scenario is outside the scope of this paper. For instance, routers with congested network link should be replaced with PRs with higher priorities. The idea of gradual deployment of protocol specific router is also introduced in [27], which implements forwarding router gradually. In conjunction with the deployment of forwarding router, the functionality of proxy router can be deployed as well.

\section{Comparison with Other Multicast Mobility Schemes}

TABLE I

COMPARISON OF MULTICAST MOBILITY SCHEMES

\begin{tabular}{l|l|l|l}
\hline \hline & MIP-BS & MIP-RS & proposal \\
\hline Modification entities & HA, AR & AR & AR, PR \\
\hline Protocol overhead & Yes & No & Yes \\
\hline Delivery overhead & Yes & No & No \\
\hline Multicast routing & Suboptimal & Optimal & Optimal \\
\hline Disruption & No & Significant & Very Little \\
\hline
\end{tabular}

We examine the multicast source handover schemes from two main perspectives: how easily they can be integrated with existing mechanisms and how efficient they are. Regarding interoperability, modified entities criteria in Table I shows the location of required modifications to existing host protocol software. Regarding performance, protocol overhead and delivery overhead show whether additional protocol and data transmissions are required over standard multicasting. Multicast routing compares each approach with standard routing. 
Under all criteria, MIP-BS is no better than the proposed scheme. A trade-off exists among ease of application and efficiency in terms of service disruption. MIP-RS will be used by applications that are insensitive to temporal service disruption while the proposed scheme will be used by applications sensitive to that.

\section{E. Scalability}

In this section, we examine the scalability from the viewpoint of traffic concentration and the state information scales.

From the viewpoint of traffic concentration, different from CBT-based multicast routing protocol such as CBT, PIM-SM enjoys the benefit of SPT that achieves network load balancing since it makes separate trees for all sources. When multicast source hands over, packets are sent via HA as is described in MIP-BT. That causes network traffic concentration around ARs temporarily. By considering the fact tremendous amount of MNs are expected to join the Internet in the forthcoming ubiquitous network, the traffic concentration around AR will be the bottle-neck of the network performance. By utilizing the proposed scheme, packets sent from $\mathrm{MN}$ are not required to traverse to ARs and eliminates or reduces temporal redundant routing. Hence, we conclude the proposed scheme can avoid traffic concentration.

From the viewpoint of state information scales, it is usually said that SPT requires multicast routers to maintain complex state information. PIM-SM routers with SPT maintains the state information ( $\mathrm{S}, \mathrm{G}$, iif, oifs), where iif denotes input interface while oif denotes output interface. Hence, the size of multicast tree state information scales $O(G * S)$. In case of the proposed scheme, a multicast router is required to handle the same state information as the PIM-SM case, hence its state information scales $O(G * S)$. The PR, on the other hand, is required to handle the state information $\left(\mathrm{AR}_{i+1}, \mathrm{AR}_{i}\right)$ as well as the one $\left(\mathrm{AR}_{i}, \mathrm{G}\right.$, iif, oif). Since one $\mathrm{AR}_{i+1}$ corresponds to one $\mathrm{AR}_{i}$, its state information scales still $O(G * S)$ though it is more complicated than that of multicast router. Hence, we conclude the proposed scheme has almost the same state information scalability level as normal SPT-based multicasting protocol.

\section{Simulation EXPERIMENTS}

In this section, we evaluate our proposed scheme from the viewpoint of routing distance and network bandwidth consumption. Routing distance is an important factor since it affects the communication delay while network bandwidth consumption is also very important factor since it affects the capacity of the network. The simulation network consists of $5 \times 5$ ARs described in Fig. 7 that is derived based on Prim's algorithm and is utilized in [17]. For this simulation experiments, we prepared time-discrete model. Each AR has its own local area network with single AP for MNs and supports our proposed protocol. In this simulation, we compared our proposed scheme with MSSMS. Since our proposed scheme is focused on handover, in this simulation, the source is moving to another network randomly in each two seconds.

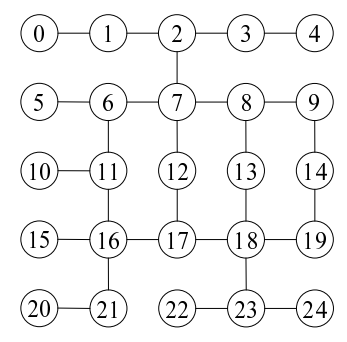

Fig. 7. Simulation Topology

Firstly, we evaluate the routing distance from source to receivers in terms of hop counts during the process of handover. We define routing gain $r_{g}$ in equation (1). Here, $N$ denotes the number of receivers, and Hcnt $t_{c o n v}$ denotes the routing hop count from source to receive with conventional scheme whilst Hcnt $_{\text {prop }}$ denotes the one with proposed scheme.

$$
r_{g}=\frac{\sum^{N}\left(H c n t_{c o n v}-H c n t_{\text {prop }}\right)}{N}
$$

Figure 8 shows the $r_{g}$ in several cases: (a) denotes the case multicast receivers are located very sparsely, i.e. Node 0 , Node 20, and Node 22 are the multicast receivers; (b) denotes the case multicast receivers are locate very densely, i.e. Node 6 , Node 7, and Node 8 are the multicast receivers; (c) denotes the case between (a) and (b), i.e. Node 1, Node 4, and Node 16 are the multicast receivers. X-axis shows the time after the simulation starts whilst Y-axis shows the routing gain $r_{g}$. As can be seen, the proposed scheme has positive routing gains over MSSMS. In Fig.8, there are sometimes handovers without any routing gain, i.e. $r_{g}=0$. These situations occur when the MN moves to new network whose AR is topologically one hop away from $\mathrm{AR}_{i}$ and is still not joining multicast tree. It is natural since all the packets are anyway going through the $\mathrm{AR}_{i}$ regardless of proposed scheme or conventional scheme. Although the routing gain $r_{g}$ is dependent on the network topology, if the network is getting more sparse, the probability causing no improvement for routing distance, i.e. $r_{g}=0$, is getting smaller.

Secondly, we evaluate the network bandwidth consumption during the process of handover. We define network bandwidth consumption as the sum of bitrate over all the links in the simulation network. Figure 9 shows the comparison between MSSMS and proposed scheme from the viewpoint of network bandwidth consumption: (a) denotes the case multicast receivers are located very sparsely, i.e. Node 0 , Node 20, and Node 22 are the multicast receivers; (b) denotes the case multicast receivers are locate very densely, i.e. Node 6, Node 7 , and Node 8 are the multicast receivers; (c) denotes the case between (a) and (b), i.e. Node 1, Node 4, and Node 16 are the multicast receivers. $\mathrm{X}$-axis shows the time after the simulation starts whilst Y-axis shows the bandwidth gain. As can be seen, the proposed scheme has gains over conventional scheme. Naturally, the value of network bandwidth consump- 


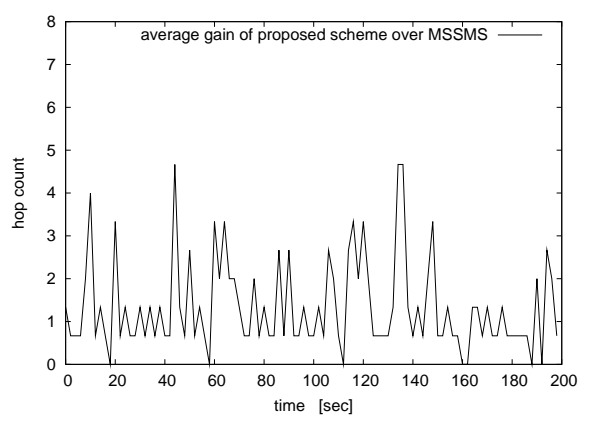

(a) Receivers $=0,20,22$

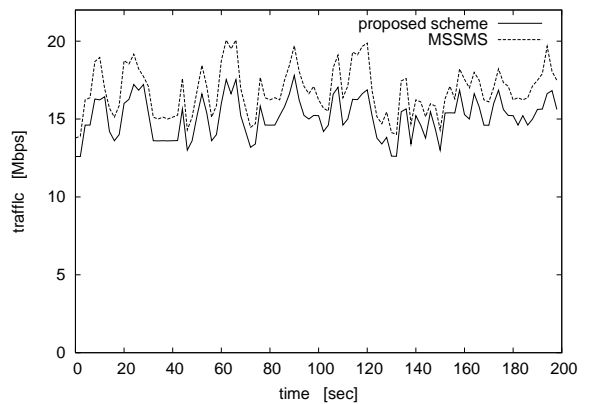

(a) Receivers $=0,20,22$

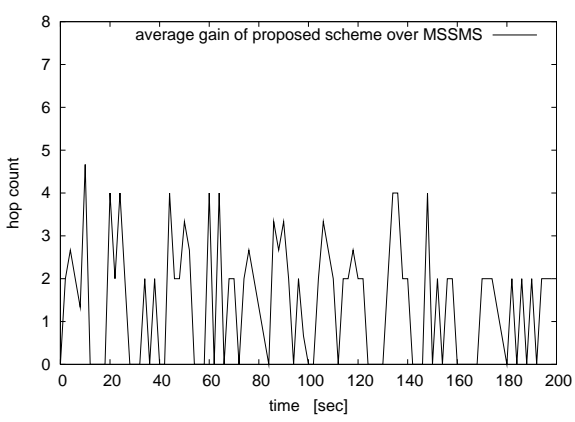

(b) Receivers $=6,7,8$

Fig. 8. Routing gain

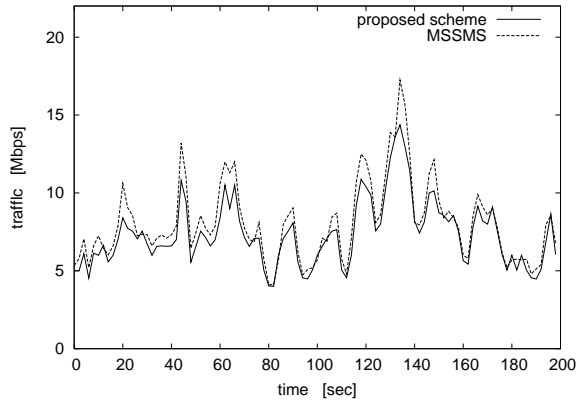

(b) Receivers $=6,7,8$

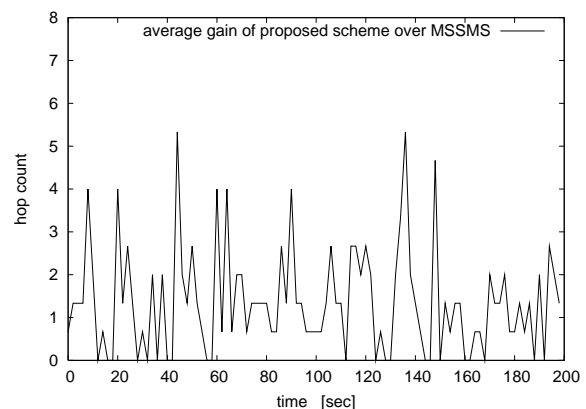

(c) Receivers $=1,4,16$

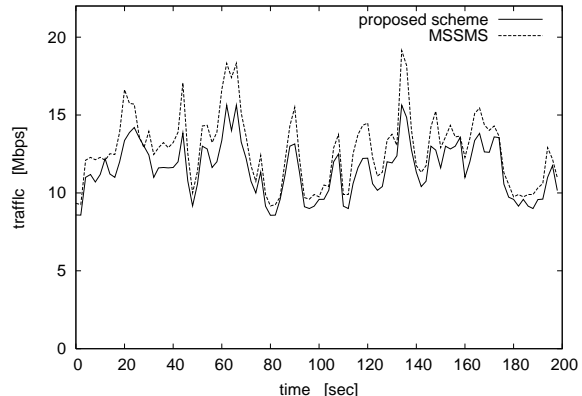

(c) Receivers $=1,4,16$

Fig. 9. Network bandwidth consumption comparison

tion is higher if the receivers are sparsely located since they cannot share the same link for multicast forwarding. However, by observing the difference between proposed scheme and MSSMS, our proposed scheme achieved better performance from the viewpoint of network bandwidth consumption if the receivers located more sparsely.

As can be seen, proposed scheme has advantages over MSSMS from the viewpoint of routing distance and network bandwidth consumption.

\section{CONCLUSION}

To support multicast source mobility over Mobile IP networks, we proposed new scheme that provides seamless handover with the help of proxy router, which assists handover by swapping network addresses and conducting packet forwarding. We examined the deployment issues of the proposed scheme over the Internet and evaluated our proposed scheme from the viewpoint of routing distance and network bandwidth consumption through simulation experiments with various parameters and clarified the effectiveness and efficiency of our proposed scheme. As our future work, we will perform further simulations and then implement our protocol over
Linux environment and evaluate the overhead of the proposed scheme.

\section{REFERENCES}

[1] D. B. Johnson, C. E. Perkins, and J. Arkko, "Mobility Support in IPv6," IETF RFC 3775, June 2004

[2] J. Arkko, V. Devarapalli, and F. Dupont, "Using IPsec to Protect Mobile IPv6 Signaling Between Mobile Nodes and Home Agents," IETF RFC 3776, June 2004.

[3] S. Deering and R. Hinden, "Internet Protocol, Version 6 (IPv6) Specification," IETF RFC 2460, Dec. 1998.

[4] N. Montavont and T. Noel, "Handover Management for Mobile Nodes in IPv6 Networks," IEEE Communications Magazine, vol. 40, no. 8, pp. 44-53, Aug. 2002.

[5] D.Waitzman, C.Partridge, and S.Deering, "Distance Vector Multicast Routing Protocol," IETF Request For Comments: 1075, Nov. 1988.

[6] J. Moy, "Multicast Extensions to OSPF," IETF Request for Comments: 1584, Mar. 1994.

[7] A. Ballardie, "Core Based Trees (CBT version 2) Multicast Routing," IETF Request for Comments: 2189, Sept. 1997.

[8] B. Fenner, M. Handley, H. Holbrook, and I. Kouvelas, "Protocol Independent Multicast - Sparse Mode (PIM-SM) : Protocol Specification (Revised)," IETF Internet Draft, Oct. 2003.

[9] W.-T. Kim and Y.-J. Park, "Scalable QoS-Based IP Multicst Over LabelSwitching Wireless ATM Networks," IEEE Network, Sept. 2000.

[10] R. Koodli, "Fast Handovers for Mobile IPv6," IETF Internet Draft (draftietf-mipshop-fast-mipv6-02.txt), July 2004. 
[11] T. G. Harrison, C. L. Williamson, W. L. Mackrell, and R. B. Bunt, "Mobile multicast (MoM) protocol : multicast support for mobile hosts," Proceedings of ACM/IEEE International Conference on Mobile Computing and Networking, 1997.

[12] J.-R. Lai, W. Liao, M.-Y. Jiang, and C.-A. Ke, "Mobile multicast with routing optimization for recipient mobility," Proceedings of IEEE International Conference on Communications, 2001.

[13] H. Omar, T. Saadawi, and M. Lee, "Multicast with reliable delivery support in the regional Mobile-IP environment," Proceedings of IEEE International Symposium on Computers and Communications, July 2001.

[14] I.-C. Chang and K.-S. Huang, "Synchronized multimedia multicast on mobile IP networks," Proceedings of IEEE International Conference on Communications, May 2003.

[15] Y.-J. Suh, D.-H. kwon, and W.-J. Kim, "Multicast routing by mobility prediction for mobile hosts," Proceedings of IEEE International Conference on Communications, May 2003.

[16] C. R. Lin and K.-M. Wang, "Mobile multicast support in IP networks," INFOCOM, 2000.

[17] C. Lin, "Mobile multicast support in IP networks," Proceedings of IEEE Global Telecommunications Conference, 2002.

[18] T. Takahashi, K. Asatani, and H. Tominaga, "Multicast Receiver Mobility over Mobile IP Networks based on Forwarding Router Discovery," Proceedings of International Conference on Networking, Apr. 2005.

[19] H.Gossain, S.Kamat, and D.P.Agrawal, "A framework for handling multicast source movement over mobile IP," Proceedings of IEEE International Conference on Communications, Apr. 2002.

[20] K. Sato, M. Katsumoto, and T. Miki, "A New Multicast Technique Supporting Source Mobility for Future Vision Delivery Service," Proceedings of International Conference on Advanced Information Networking and Application, 2004.

[21] C.S.Jelger and T. Noel, "Supporting mobile SSM sources for IPv6," Proceedings of IEEE Global Telecommunications Conference, 2002.

[22] W. Jia, W. Zhou, and J. Kaiser, "Efficient algorithm for mobile multicast using anycast group," IEE Proceedings - Communications, vol. 148, no. 1, Feb. 2001.

[23] Z. D. Shelby, D. Gatzounas, A. Campbell, and C.-Y. Wan, "Cellular IPv6," IETF Internet Draft (draft-shelby-seamoby-cellularipv6-00.txt), Nov. 2000.

[24] S. Bhattacharyya, "An Overview of Source-Specific Multicast (SSM)," RFC 3569, July 2003.

[25] T. Narten, E. Nordmark, and W. Simpson, "Neighbor Discovery for IP Version 6 (IPv6)," IETF RFC 2461, 1998.

[26] G. Xylomenos and G. C. Polyzos, "IP Multicast for Mobile Hosts," IEEE Communications Magazine, Jan. 1997.

[27] T. Takahashi, J. Harju, K. Asatani, and H. Tominaga, "A routing-aware handover scheme for mobile ip," Proceedings of IEEE International Conference on Communications, May 2005. 